BULLETIN OF THE

AMERICAN MATHEMATICAL SOCIETY

Volume 80, Number 4, July 1974

\title{
SQUARE-INTEGRABLE KERNELS AND GROWTH ESTIMATES FOR THEIR SINGULAR VALUES
}

\author{
BY JAMES ALAN COCHRAN
}

Communicated by A. S. Householder, October 22, 1973

Let $K(x, y), 0 \leqq x, y \leqq \pi$, be Lebesgue square-integrable. Define

$$
K^{(r)}(x, y) \equiv \partial^{r} K(x, y) / \partial x^{r} \quad(r=0,1,2, \cdots, s)
$$

for nonnegative integer $s$, and assume that $K(x, y)$ is extended, as an even function of $x$ if $s$ is even, and as an odd function of $x$ if $s$ is odd, into the domain $-\pi \leqq x \leqq 0$, and thence as a periodic function of $x$ with period $2 \pi$. Let the singular values $\mu_{n}$, where

$$
\begin{aligned}
\phi_{n}(x) & =\mu_{n} \int_{0}^{\pi} K(x, y) \Psi_{n}(y) d y, \\
\Psi_{n}(x) & =\mu_{n} \int_{0}^{\pi} \overline{K(y, x)} \phi_{n}(y) d y
\end{aligned}
$$

with $\left\|\phi_{n}\right\|,\left\|\Psi_{n}\right\| \neq 0$, be ordered (indexed) in the natural manner according to increasing size, namely $0<\mu_{1} \leqq \mu_{2} \leqq \mu_{3} \leqq \cdots$.

In a perhaps overlooked paper, Smithies [8] has shown that

THEOREM 1. If the $K^{(r)}(x, y), 0 \leqq r \leqq s-1$, are continuous in $x$, a.e. in $y$, and $K^{(s)}(x, y)$ is in $\mathscr{L}^{p}(x)$, a.e. in $y$, for some $1<p \leqq 2$, then

$$
\int_{0}^{\pi}\left[\int_{0}^{\pi}\left|K^{(s)}(x+h, y)-K^{(s)}(x-h, y)\right|^{p} d x\right]^{2 / p} d y \leqq C|h|^{2 \alpha}
$$

for constant $C$, where $\alpha>0$ if $s>0, \alpha>(2-p) / 2 p$ if $s=0$, implies

$$
1 / \mu_{n}=O(1) / n^{\alpha+s+1-1 / p} \quad \text { as } n \rightarrow \infty \text {. }
$$

Recognizing (1) as essentially an integrated Lipschitz condition, and using various properties associated with the class of kernels which satisfy such a condition, we can substantially generalize the above result.

We say that $K^{(s)}(x, y)$ is in Lip $\alpha$ if

$$
\left|K^{(s)}(x+h, y)-K^{(s)}(x-h, y)\right|<|h|^{\alpha} A(y) \quad(0<\alpha \leqq 1)
$$

where $A(y)$ is nonnegative and square-integrable. Likewise $K^{(s)}(x, y)$ is said to be (relatively uniformly) of bounded variation if for all $N \geqq 1$ and

AMS (MOS) subject classifications (1970). Primary 45H05, 45M05; Secondary 47B10, 47A10. 
arbitrary choice of partition $0 \leqq x_{0} \leqq x_{1} \leqq \cdots \leqq x_{N} \leqq \pi$,

$$
\sum_{n=1}^{N}\left|K^{(s)}\left(x_{n}, y\right)-K^{(s)}\left(x_{n-1}, y\right)\right|<B(y)
$$

where $B(y)$ also is nonnegative and square-integrable. More generally, we say that $K^{(s)}(x, y)$ belongs to $\operatorname{Lip}(\alpha, p)$ if

$$
\int_{0}^{\pi}\left|K^{(s)}(x+h, y)-K^{(s)}(x-h, y)\right|^{p} d x<|h|^{\alpha p} A^{p}(y) \quad(0<\alpha \leqq 1)
$$

with $\mathscr{L}^{2} A \geqq 0$.

Analogous to the one-variable situation (see Hardy and Littlewood [5], [6], for example), we may establish

Property 1. Kernels in $\operatorname{Lip}(\alpha, p)$ also belong to $\operatorname{Lip}(\alpha, q)$ for all $1 \leqq q<p$. Kernels in $\operatorname{Lip} \alpha$ are automatically in $\operatorname{Lip}(\alpha, p)$ for all $p \geqq 1$.

Property 2. Kernels which are (relatively uniformly) of bounded variation belong to $\operatorname{Lip}(1,1)$.

Property 3. If $K(x, y)$ is absolutely continuous in $x$, a.e. in $y$, and

$$
\int_{0}^{\pi}\left[\int_{0}^{\pi}\left|K^{(1)}(x, y)\right|^{p} d x\right]^{2 / p} d y<\infty, \quad p>1,
$$

then $K(x, y)$ is in $\operatorname{Lip}(1, p)$.

Property 4. If a kernel belongs both to $\operatorname{Lip}(\alpha, p)$ and to $\operatorname{Lip}(\beta, q)$ with $p<q$, then it belongs to $\operatorname{Lip}(\gamma, r)$ for all $p \leqq r \leqq q$, where

$$
\gamma=\alpha \frac{p(q-r)}{r(q-p)}+\beta \frac{q(r-p)}{r(q-p)} .
$$

Property 1 permits the immediate extension of Theorem 1 to the case $p>2$ with the resultant growth estimate

$$
1 / \mu_{n}=O(1) / n^{\alpha+s+1 / 2} \quad \text { as } n \rightarrow \infty .
$$

In view of Property 3 , moreover, we have essentially the generalization for arbitrary $p>1$ of a result originally established for $p=2$ by Chang [1].

One of the more general results which can be established using the "convexity" Property 4 is

THEOREM 2. Let $K(x, y)$ satisfy the hypotheses of Theorem 1 for some nonnegative integer $s$ and $p>1$. If $K^{(s)}(x, y)$ belongs both to $\operatorname{Lip}(\alpha, p)$ and to $\operatorname{Lip}(\beta, q)$, with $p<q$, then $1 / \mu_{n}=O(1) n^{-\sigma}$ as $n \rightarrow \infty$ where

(i) for $q \leqq 2$,

$$
\begin{aligned}
\sigma & =\alpha+s+1-1 / p, & & p q(\alpha-\beta)>q-p, \\
& =\beta+s+1-1 / q, & & p q(\alpha-\beta) \leqq q-p,
\end{aligned}
$$


(ii) for $p \leqq 2<q$,

$$
\begin{array}{rlrl}
\sigma & =\alpha+s+1-1 / p, & p q(\alpha-\beta)>q-p, \\
& =\frac{q(2 \beta+\alpha p+2 s+1)-p(2 \alpha+\beta q+2 s+1)}{2(q-p)}, \\
& =\beta+s+1 / 2, & 0<p q(\alpha-\beta) & \leqq q-p, \\
& \alpha \leqq \beta,
\end{array}
$$$$
\text { (iii) and for } p>2 \text {, }
$$

$$
\begin{aligned}
\sigma & =\alpha+s+1 / 2, & & \alpha>\beta, \\
& =\beta+s+1 / 2, & & \alpha \leqq \beta .
\end{aligned}
$$

When $s=0$, the additional provisos $\alpha+1 / 2>1 / p$ and $\beta+1 / 2>1 / q$ may be needed.

In view of the several properties above, Theorem 2 contains as special cases the generalizations of other recent results of the author [3]. Furthermore, since they pertain to the singular values, any such conclusions must perforce also extend our knowledge concerning the growth behavior of the characteristic values of "smooth" kernels (see Hille and Tamarkin [7], Cochran [2], for example).

The relationship of the above theorems and their sundry special cases to summability results for the classical Fourier coefficients of integrable functions of a single real variable satisfying various smoothness criteria will be explored at length elsewhere [4].

\section{REFERENCES}

1. S. H. Chang, A generalization of a theorem of Hille and Tamarkin with applications, Proc. London Math. Soc. (3) 2 (1952), 22-29. MR 13, 950.

2. J. A. Cochran, The analysis of linear integral equations, McGraw-Hill, New York, 1972.

3. - The nuclearity of operators generated by Hölder continuous kernels, Proc. Cambridge Philos. Soc. (1974)

4. - Summability of singular values of $\mathscr{L}^{2}$ kernels-analogies with Fourier series (to appear).

5. G. H. Hardy and J. E. Littlewood, Some properties of fractional integrals. I, Math. Z. 27 (1928), 565-606.

6. - Notes on the theory of series (IX): On the absolute convergence of Fourier series, J. London Math. Soc. 3 (1928), 250-253.

7. E. Hille and J.D. Tamarkin, On the characteristic values of linear integral equations, Acta Math. 57 (1931), 1-76.

8. F. Smithies, The eigen-values and singular values of integral equations, Proc. London Math. Soc. (2) 43 (1937), 255-279.

Department of Mathematics, Virginia Polytechnic Institute and State UniVERSITY, BLACKSBURG, VIRGINIA 24061 